\title{
Systems Approach to Cultural Tourism and Events
}

\author{
Tadeja Jere Jakulin \\ University of Primorska, Faculty of Tourism Studies - Turistica, Slovenia \\ tadeja.jerejakulin@upr.si
}

In this paper, we will present the systems approach methodology, which has been widely used in the natural sciences and engineering for the last seven decades since Ludwig von Bertalanffy published his manifesto on general systems theory (1952) and Norbert Wiener his on cybernetics (1948). The intention of general systems theory and cybernetics is the 'ontology' of action, which is shown by feedback information. Its goal is to find a method to predict the consequence of a decision-making action. Industrial engineering recognised it when Forrester published Industrial Dynamics (1961), and social sciences rediscovered it with Senge's work on the learning organisation The Fifth Discipline (1990). Cultural tourism is an element of a complex tourism system within global and local dimensions, in which cultural events, as even smaller elements, play essential roles within the range of tourism goods and services. As a methodology for the research of complex phenomena, the systems approach will explain two of its methods, systems thinking and modelling, as those that can significantly influence decision-making when taken into account. We will show the appropriateness of the methodology within cultural tourism decision-making and modelling.

Keywords: systems approach, analytical and systems thinking, complexity, cultural tourism and events, modelling https://doi.org/10.26493/2335-4194.12.185-191

\section{Introduction}

The systems approach became an optimal methodology for creating solutions within engineering and the natural sciences a few decades ago. The feedback loop, which represents the circle within a system, shows that correct anticipation of the future outcome is possible only if a decision-maker observes problems in reality from a systems point of view. Such a point of view, or 'the big picture,' is a view in which all observed subjects or objects are seen as connected, interdependent, influencing each other, as is known in nature and engineering. Frequently, it is difficult to see closed connections and interdependency within social and organisational systems composed of elements (components, departments) that are nested within each other even though they are not only elements but also sys- tems. Nesting and the difficulties of seeing boundaries among the elements make them complex systems and, as such, they need appropriate methodology to explain them, as well as solutions for their problems. Tourism and its subsystems of cultural tourism with events and all their connections, boundaries, and interdependency represent systems complexity. The systems approach, with its systems thinking and modelling methods, can survive the most rigid testing and can find systems solutions.

\section{Analytical and Systems Thinking}

A vision without systems thinking ends up painting lovely pictures of the future with no deep understanding of the forces that must be mastered to move from here to there (Senge, 1990). 


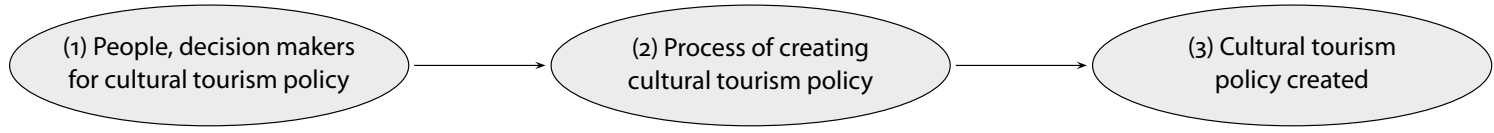

Figure 1 Model of Conventional (Linear) Thinking



Figure 2 Systems Model of Cultural Tourism Policy

Some relevant paradigms to analysis were described by Rosenhead (1989) and Mulej et al. (1994), including soft analysis, the hard study of a system, critical thinking, strategic options development and analysis, and the dialectical theory of systems. The linear (analytical) approach is based on interpretation as a three-step thought process. It takes apart that which it seeks to understand, then attempts to explain the behaviour of the parts taken separately, and finally, and finally tries to develop an aggregate understanding of the components into an explanation of the whole.

The systems approach uses a different process. It puts the system in the context of the larger environment of which it is a part and studies the role it plays in the larger whole. The parts are no longer the primary focus. The elements are essential, but what is more important is the interrelationship between the elements as they work together to fulfil the purpose of the whole system. A systems approach is optimal for understanding interdependency, which requires a way of thinking differently than analysis; it needs systems thinking. The number of works dedicated to the systems thinking, systems models, and methodologies devoted to social, economic and natural sciences is enormous. These include System Dynamics (Forester, 1961), System Thinking (Senge, 1990; Vennix, 1996), Autopoietic System (Maturana \& Varela, 1998), Living Systems (Miller, 1978), Viable Systems (Beer, 1959), Anticipatory Systems (Rosen, 1985) as well as others. Systems thinking searches for the optimal solutions and answers from right to the left, as presented with the systems model of cultural tourism policy in Figure 2.

The primary step of the system approach starts at A: the outputs or vision of the optimal cultural tourism policy. The expert group uses as fundamental questions the questions about the influence of optimal cultural tourism (outputs, A) to the environment (E other people, nature, community), uses feedback information ( $\mathrm{B}$ - what will cultural tourism policy bring to the $\mathrm{E}$ ) and asks 1 . What will the vision (A), outputs, organisational effectiveness, environmental effectiveness (Getz \& Frisby, 1988) bring to the environment, environmental uncertainty, community context, networks complexity (E)? and 2. What is the current situation (C), inputs, ideas, teams, finances, material resources, facilities (Getz \& Frisby, 1988) for achieving (A)? and 3. How can they help in the process (B) either with the help or without any worries if they cannot influence the process? To avoid the trap of the simplicity of systems thinking, one can build a simulation model of effective decision-making in which one attempts to implement the optimal systems solutions. The model helps identify the flows and uses of resources, including money and information, which are the life-blood of all events. It helps identify key stakeholders who have to be brought into decision-making (Getz, 2004). Systems evaluation helps the manager keep the 'big picture' in mind, whereas the natural tendency is to get lost in daily problem-solving.

The systems model differs from the linear model. Environment and feedback information are those parts of systems thinking and modelling that enable see- 


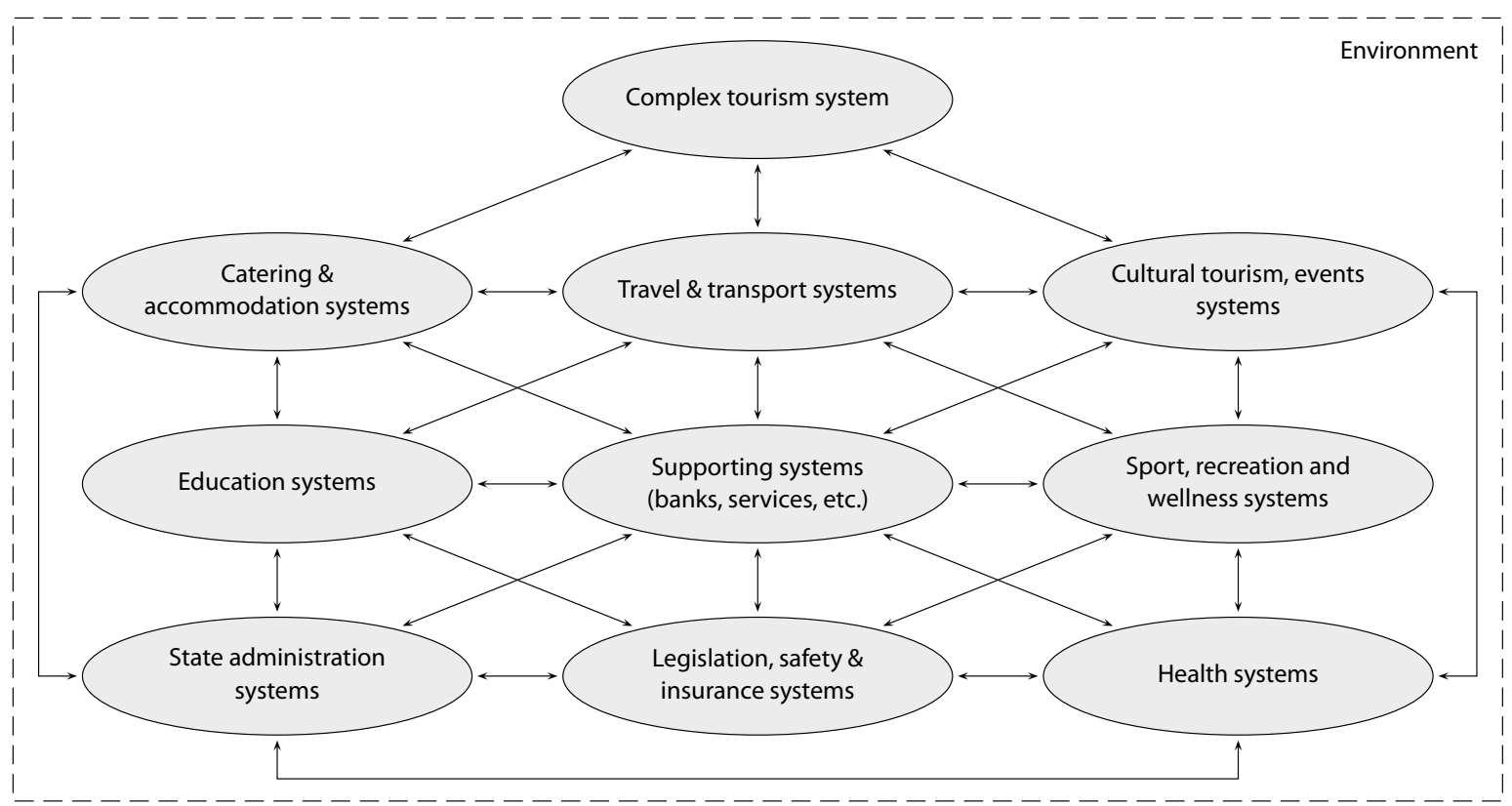

Figure 3 Interdependency of the Cultural Tourism System, Other Subsystems and the Environment

ing the 'big picture' point of view to an observer. The model's feedback information shows internal evaluation, which pertains to management's collective responsibility to ensure that all functions and programmes are meeting goals and that operations are as efficient as possible. The external evaluation covers both the organisation's linkages with other agencies and stakeholders and the impacts of its actions (Getz, 2004)

Ongoing assessment helps steer the institution, organisation, and manager towards meeting their goals. The event can always be improved, marketing enhanced, and benefits increased. Furthermore, evaluation can help avoid and suggest ways to eliminate or enhance negative consequences. Evaluation means assigning a value or worth to something, and it inherently involves subjective judgment as well as technical analysis (Getz, 2004).

\section{The Complexity of Cultural Tourism and Events}

The art of systems thinking lies in seeing through complexity to the underlying structures generating change (Senge, 1990).

In the context of tourism, we can use an expanded concept of culture. From this point of view, culture serves not only human education through the provision of music, theatre, museums, exhibitions, festivals and listed buildings, but permeates every area of human life (Dreyer, 200o). According to Adams (2008), cultural tourism is a type of special interest tourism involving leisure travel to view or experience the distinctive character of a place, its peoples, and its products or productions. The complexity of cultural tourism and events is visible since they are elements of a more extensive, tourism system. The word 'complex' is used only to indicate that the problem treated here cannot be expressed solely in hard (quantitative) relations and that most relevant values are qualitative. We consider complex systems to be networks created of many components, which interact among each other in a nonlinear manner; they may evolve through self-organisation so that they are neither completely regular nor completely random (Sayama, 2015). With a conception of complex systems, we also present a system within which the complexity of interactions among system elements plays a leading role. These elements are systems themselves and, for this reason, the behaviour of a system as a whole can hardly be pre- 
dicted: the system of systems, which exchanges energy and information with its environment while in transit, inflected by internal and external influences. Organisational systems are complex because of the existing relations and nesting of its subsystems. This is represented in Figure 3.

The systems within a system of tourism nest within each other, which means that they represent subsystems at the same time that they represent systems as wholes. The interdependency and relations among the entities of these subsystems are far more critical than independent systems. This is especially seen between the subsystems of travel and transport, catering and accommodation, art and culture, sports, recreation and wellness subsystems. There are certain interdependent relationships among all subsystems, which strongly influence each other. If we map the tourism system to the local (national) or global (international destination), we reach a level of a system, which encompasses a wide variety of partners, branches and institutions. They create a complex system as such; with all interconnections, interdependency, and nesting in each other (dependent on the size of a subsystem).

\section{General Tourism System Model}

Remember, always, that everything you know, and everything everyone knows, is only a model. Get your model out there where it can be viewed. Invite others to challenge your assumptions and add their own (Meadows, 2008).

A society is a real world, which changes by altering relations among its participants as well as interactions with the environment. Tourism is often studied as an industry, alongside hospitality, with related educational programmes stressing professional career preparation. It is also a social, cultural, and economic phenomenon with all its potential impacts (Getz, 2007). Learning and experience through decision-making provide tourism development and growth that are observed through its evolution. The evolution of society and skills as part of the past and the anticipation of the future causes these systems to grow and develop with the environment as the restriction. Thus, we can say that tourism system is, as its subsystems, dynamic. Regulation is necessary but far away

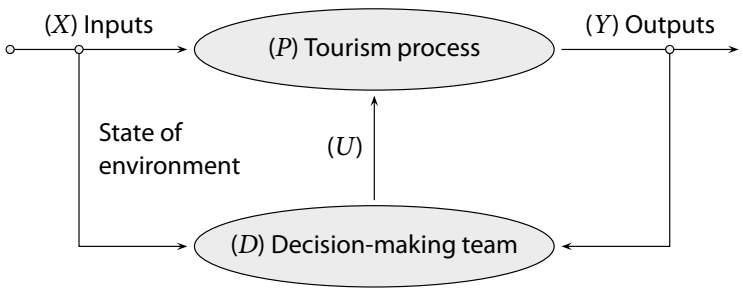

Figure 4 A General Model of Tourism as a Goal-Oriented System (adapted from Jere Jakulin \& Kljajić, 2006)

from being sufficient. The most important is a strategic vision of a development, and the understanding that the environment influences the prediction of the strategic vision.

For this reason, tourism systems can be defined slightly differently, so that the fundamental causes of system behaviour are emphasised. Usually, they are called 'management subsystems.' The tourism system can be described with a model, which is an idealised and simplified image of the real situation or phenomenon and contains only necessary quantities and their functional dependencies. The model is an attempt to identify critical variables in a case study and the relationship that exists among them (Kljajić, 1998).

In Figure 4, we define a general model of tourism as a goal-oriented system with a pair $(P, D) . P$ represents the managing process in the tourism system, $D$ the managing subsystem. Loop $P \rightarrow Y \rightarrow D \rightarrow U \rightarrow P$ represents feedback information, which functions on the cause-and-effect principle; therefore, we can call it 'reactive control.' For small perturbances, such control suffices. For decision making in a tourism system, information from the environment is necessary. The chain $X \rightarrow D \rightarrow U \rightarrow P$ provides feed-forward data, which represents the anticipation of the future state of the situation. It is an essential part of the strategy of goal-oriented systems.

The inputs consist of ideas, scenarios, tangible resources, human resources, which start the process of cultural tourism policy creation and outputs, which can also include unintended and negative impacts that should be identified through research. The decisionmaking team plays a role in the model evaluation and 
consists of those experts and people who create goals and have responsibility for the system's development. The team and its capabilities make the systems work towards their balanced functioning and goals.

\section{Building a Causal Loop Diagram Model}

Modelling is a part of systems methodology, which requires more than basic knowledge of the topic one models. By modelling, we understand an activity enabling us to describe our experiences within a specific procedure (mental model) with one of the existing languages in the framework of a precise theory (Jere Jakulin \& Kljajić, 2006). Model building in tourism seeks to understand a complex relationship and to aid the management of the place or process (Jafari, 2000). From a pragmatic point of view, a system is defined by the double $S=(E, R)$, where $e_{i} \in E \subset$ $U=1,2, \ldots n$ represents the set of elements, $R \subseteq E \times E$ the relation between the elements, and the $U$ universal set. The construction of concrete systems requires specific knowledge $K\left(e_{i}\right) \in E$ (property of elements) to identify the elements of the systems (including those from environments) and a theory $T\left(e_{i}, e_{j}\right) \subset R$ to find relationships among the elements. Each element $e_{i}$ can be set as well as $R_{j} \in R, j=1,2, \ldots m$ defining different relations between the elements. Such a procedure is inductive and represents the model of a real system. In other words, modelling represents the activity of describing our experiences by using one of the existing languages within the framework of a specific theory such as formal concepts analysis described in (Wolff, 1999). In this way, our experiences also become accessible to others in that they may be proven, confirmed, rejected, broadened or generalised.

From a causal loop diagram, shown in Figure 5, one can derive that there is one basic circle (-) of the causal loop, which means the growth of tourists' number and borders of growth, caused by infrastructure and diminished environment attraction. In a vision of cultural tourism policy, we have to predict development as a whole to avoid limitations. If in the reinforcement circle, which consists of investments, tourism infrastructure, environment attraction, only one element starts to fall (-), this means all of the other elements will fall.

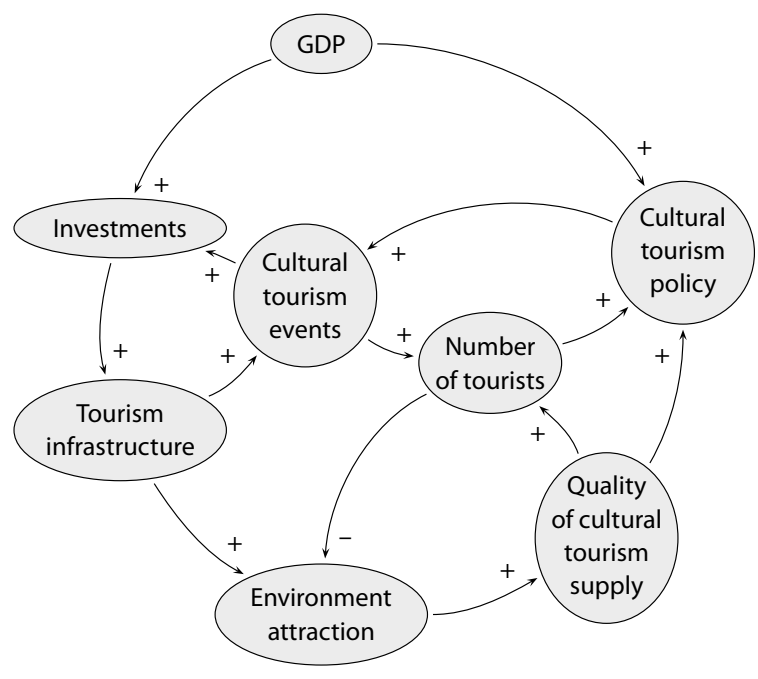

Figure 5 CLD Model of the Interdependence of Cultural Tourism Policy Elements

The above-discussed model (Figure 5) requires decision-making given by a group of experts and decision-makers who influence areas of cultural tourism. Figure 5 presents the cultural tourism expert group as a part of the cultural tourism legislative process, in which modelling and ideas about penal code determination represent a knowledge-capturing process in the form of the structure and behaviour of the model. Once the model is defined and validated, experimentation with different scenarios is possible. The cultural tourism expert group determines the set of different ideas, which represents possible future activities in the real system. The results gathered as the output of the model are evaluated with the multi-criteria evaluation function. At this stage, many different multicriterial evaluation methods may be used, from the weighted average (Vincke, 1992) to the Analytical Hierarchy Process (A HP), (Saaty, 2012) and Expert systems (es) (Rajkovič \& Bohanec, 1991). Information feedback provides the expert group with the possibility of creatively determining a new set of ideas on cultural tourism policy and multi-criteria evaluation functions relating to the given situation. Simulated and actual performances of the system are compared to adapt the strategy according to changes in the environment.

The systems thinking solving method with sim- 


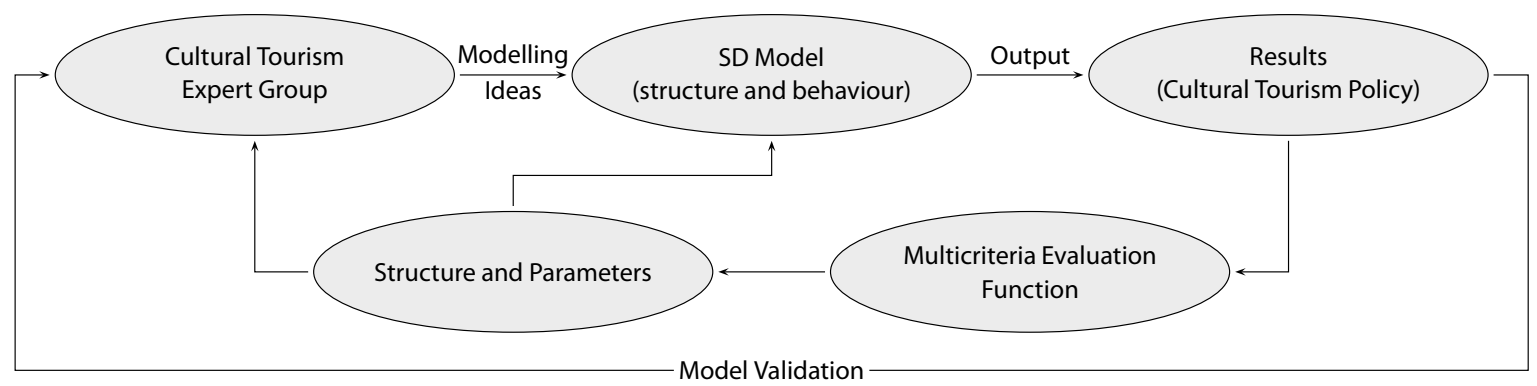

Figure 6 The Principle Scheme of Simulation Methodology for Decision-Making Support (adapted from Jere Jakulin \& Kljajić, 2006)

ulation model follows standard steps: state analysis, development of causal-loop diagrams, writing of the model's equations, and model implementation. Particular scenarios that form and determine a tourist market in a particular environment are tested on a simulation system. A simulator is connected to the GSs (Group Support System). The participants using Gss work directly with the system simulator. A system simulator is connected to a database, which is necessary for simulation model activation. Simulation results are evaluated both with the group decision-making support system and with expert systems. In all of this, the understanding of the system increases. With the described model, the experimental loop on a simulation model has been finished with the help of the system simulator and scenario ranking. The elements of the decision-making support system are Powersim, a tool for the construction and use of a simulator; Ventana Group Systems, the Ventana group working support system; DEX, a shell of an expert system expert; and Expert Choice, evaluation with the AHP method. Since the work with group decision-making tool is anonymous, it raises creative thinking, which enables a higher flow of ideas and reduces unwanted influences. The participants become more relaxed since no one knows where the ideas come from and, thus, creativity is released; this would not be the case in the more conventional ways of working. The work time decreases and the efficiency of participants increases (Jere Jakulin, 2017). The final result is better, as the decision becomes a group decision with which conflict between polarised groups is minimised, and a consensus is achieved for the development of further actions.

\section{Conclusions}

The present article discusses the method of describing and modelling the complex cultural tourism system from the systems and decision-making point of view. The systems approach has become a necessity in contemporary life, which we see as a modern complex system composed of a variety of other systems and their elements. In the paper, we discussed the cultural tourism system and its elements or subsystem of travelling and transport systems, catering and accommodation systems from a systems point of view within the environment. The cultural tourism system was described as a so-called soft system phenomenon, in which people, with their actions, knowledge, and characters play the leading roles, representing a complex system of a society. As we reach a certain level of complexity, we must search for an optimal methodology to find an optimal way of dealing with this complexity. The methods of systems dynamics, systems thinking, and modelling are some of them. Therefore, the anticipated system is much closer to describing the essence of complex systems behaviour. However, the influence of the observer in the process of modelling the complex system is of primary importance. The role of the observer usually is usually played by expert groups and decision-makers who are entitled to accept decisions regarding policies in society. In literature, this problem has not been sufficiently considered.

\section{References}

Adams, K. (2008). Cultural tourism. In W. A. Darity Jr. (Ed.), International encyclopedia of the social sciences (2nd ed., pp. 201-202). Detroit, MI: Macmillian. 
Beer, S. (1959). Cybernetics and management. London, England: English U. Press.

Bertalanffy, L. V. (1952). General system theory. New York, NY: George Braziller.

Dreyer, A. (200o). Kulturtourismus. Munich, Germany: Oldenbourg Verlag.

Forrester, J. W. (1961). Industrial dynamics. Cambridge, M A: MIT Press.

Getz, D. (2004). Event management and event tourism (2nd ed.). New York, Ny: Cognizant Communication Corporation.

Getz, D. (2007). Event studies: Theory, research and policy for planned events. Burlington, MA: Butterworth and Heinemann.

Getz, D., \& Frisby, W. (1988). Evaluating management effectiveness in community-run festivals. Journal of Travel Research, 27(1), 22-27.

Jafari, J. (Ed.) (200o). Encyclopedia of tourism. New York, NY: Routledge.

Jere Jakulin, T. (2017). Systems approach as a creative driving force for a destination. In A. Királ'ová (Ed.), Driving through creative destinations and activities (pp. 1-19). Hershey, PA: IG I Global.

Jere Jakulin, T., \& Kljajić, M. (2006). Systems approach to complex systems modelling with special regards to tourism. Kybernetes: The International Journal of Systems \& Cybernetics, 35(7/8), 1048-1058.

Kljajić, M. (1998). Modelling and understanding the complex system within cybernetics. In M. J. Ramaekers (Ed.), 15th international congress on cybernetics (pp. 864-869). Namur, Belgium: Association International de Cybernetique.

Meadows, D. H. (2008). Thinking in systems: A primer. Hartford, vт: Chelsea Green Publishing.

Miller, J. G. (1978). Living systems. New York, Ny: McGrawHill.
Maturana, R. H., \& Varela, J. F. (1998). The tree of knowledge. Bern, Switzerland: Scherrz Verlag.

Mulej, M., et al. (1994). Teorije sistemov. Maribor: University of Maribor.

Rajkovič, V., \& Bohanec, M. (1991). Decision support by knowledge explanation. In H. G. Sol \& J. Vecsenyi (Eds.), Environments for supporting decision processes (pp. 4757). Amsterdam, The Netherlands: Elsevier.

Rosen, R. (1985). Anticipatory systems. New York, NY: Pergamon Press.

Rosenhead, J. (1989). Rational analysis for a problematic world. Chichester, England: Wiley.

Saaty, T. L. (2012). Decision making for leaders: The analytic hierarchy process for decisions in a complex world. Pittsburgh, PA: Rws Publications.

Sayama, H. (2015). Introduction to the modelling and analysis of complex systems. New York, NY: State University of New York Press.

Senge, P. (1990). The fifth discipline: The art and practice of the learning organization. New York, NY: Doubleday October.

Vennix, J. A. M. (1996). Group model building: Facilitating team learning using system dynamic. Chichester, England: Wiley.

Vincke, P. (1992). Multicriteria decision-aid. Chichester, England: Wiley.

Wiener, N. (1948). Cybernetics. Chichester, England: Wiley.

Wolff, K. E. (1999). Concept, states, and systems. In D. M. Dubois (Ed.), CAsys'99 Third International Conference (pp. 233-243). Melville, NY: American Institute of Physics.

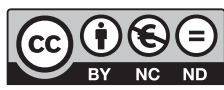

This paper is published under the terms of the Attribution- NonCommercial-NoDerivatives 4.0 International (CC BY-NC-ND 4.0) License. 